\title{
Estudo da dinâmica evolutiva do mosquito Aedes aegypti sob controle SIT e estratégia de replacement
}

\author{
Rafaela Veloso ${ }^{1}$ \\ Unifesp, São José dos Campos, SP \\ Evelyn Braga ${ }^{2}$ \\ Unifesp, São José dos Campos, SP \\ Leonardo Kanashiro 3 \\ Unifesp, São José dos Campos, SP \\ Bruno Ferrari ${ }^{4}$ \\ Unifesp, São José dos Campos, SP \\ Luiz Leduino de Salles Neto ${ }^{5}$ \\ Unifesp, São José dos Campos, SP \\ Weldon Lodwick ${ }^{6}$ \\ University of Colorado, Denver, USA \\ Margareth Capurro ${ }^{7}$ \\ USP, São Paulo, SP.
}

\begin{abstract}
Resumo. Neste trabalho foram feitas simulações da introdução de dois grupos de mosquitos Aedes aegypti transgênicos em uma região ao longo do tempo - um grupo de inseto estéril (SIT - Sterile Insect Technique) e um grupo que carrega uma alteração no DNA que leva o mosquito à morte quando em contato com o vírus da dengue. Primeiramente, simulou-se a inserção de cada grupo separadamente e, em seguida, analisaram-se os dois casos sendo aplicados em conjunto. Os modelos foram testados sob parâmetros variáveis de sazonalidade, considerando uma distribuição inicial de população selvagem fixa aproximada da região de estudo (Ribeirão Preto). Os resultados indicam quais políticas seriam eficazes para extinção do vetor na região estudada.
\end{abstract}

Palavras-chave. Modelagem Matemática, Aedes aegypti, Dengue.

\section{Introdução}

O mosquito Aedes aegypti, também conhecido como mosquito-da-dengue é transmissor dos vírus da dengue, além de zika e chikungunya. Entre essas, a dengue é considerada a doença mais grave. O Brasil, especificamente, vive uma situação alarmante em relação à dengue, conforme dados divulgados pelo Ministério da Saúde [4]: foram registrados 1.544.987 casos de dengue em 2019 (com 782 mortes), um aumento de 488\% em relação a 2018. O boletim epidemiológico $\mathrm{n}^{\mathrm{O}}$

\footnotetext{
${ }^{1}$ rafaelaveloli@gmail.com

2 evelynbraga@outlook.com

${ }^{3}$ kanashirohleonardo@gmail.com

${ }^{4}$ bjferrari12@gmail.com

${ }^{5}$ luiz.leduino@gmail.com

${ }^{6}$ wlodwick@gmail.com

${ }^{7}$ mlcapurro@gmail.com
} 
07 de 2020 aponta que o número de casos prováveis da doença cresceu $19 \%$ nas cinco primeiras semanas do ano em comparação com o mesmo período do ano passado.

Por esse motivo é essencial desenvolver métodos de controle que ajudem no combate ao mosquito transmissor. Nos últimos anos vêm sendo implementadas técnicas de combate usando o próprio mosquito, através de modificação genética. A mais conhecida é a de liberação do inseto estéril (SIT - Sterile Insect Technique) [1], que consiste na geração de mosquitos estéreis para inserção forçada no ambiente. Nesse caso, o acasalamento entre uma fêmea selvagem e um macho estéril produzirá ovos que não eclodirão, reduzindo a população ao longo do tempo. Essa estratégia é chamada supressão.

Neste trabalho, foram feitas simulações da introdução dos dois grupos de mosquitos Aedes aegypti transgênicos em uma região ao longo do tempo. Primeiramente, simulou-se a inserção de cada grupo separadamente e, em seguida, analisaram-se os dois casos sendo aplicados em conjunto. Os modelos foram testados sob parâmetros variáveis de sazonalidade, considerando uma distribuição inicial de população selvagem fixa aproximada da região de estudo (Ribeirão Preto).

\section{O Modelo Matemático}

Para a modelagem, considerou-se, além da população de mosquitos, a população de humanos, pois a taxa de transmissão da dengue pelas pessoas afeta a dinâmica populacional dos mosquitos em especial devido à morte dos mosquitos geneticamente modificados do grupo de replacement ao contrairem o vírus por picarem alguém infectado. Da mesma forma, a substituição da população de mosquitos também tem efeito sobre a ocorrência da doença entre humanos, de modo que há uma relação de dependência entre as populações que é relevante para o presente estudo.

A Tabela 1 apresenta os diferentes grupos populacionais envolvidos.

\begin{tabular}{ll}
\hline Notação & \multicolumn{1}{c}{ População } \\
\hline$A$ & Fase aquática \\
$M$ & Machos selvagens \\
$H$ & Fêmeas saudáveis não fecundadas (sem o gene replacement) \\
$F_{S}$ & Fêmeas saudáveis fecundadas por machos selvagens (sem o gene replacement) \\
$F_{I}$ & Fêmeas infectadas fecundadas por machos selvagens (sem gene replacement) \\
$N$ & Machos inférteis \\
$G_{S}$ & Fêmeas saudáveis fecundadas por machos inférteis (sem o gene replacement) \\
$G_{I}$ & Fêmeas infectadas fecundadas por machos inférteis (sem o gene replacement) \\
$F_{S}^{*}$ & Fêmeas saudáveis fecundadas por machos replacement (sem o gene replacement) \\
$F_{I}^{*}$ & Fêmeas infectadas fecundadas por machos replacement (sem o gene replacement) \\
$A_{E}$ & Fase aquática replacement \\
$M_{E}$ & Machos replacement \\
$H_{E}$ & Fêmeas não fecundadas replacement \\
$F_{E}$ & Fêmeas fecundadas replacement \\
$S_{H}$ & Humanos suscetíveis \\
$I_{H}$ & Humanos infectados \\
$R_{H}$ & Humanos recuperados \\
\hline
\end{tabular}

Tabela 1: Grupos populacionais incluídos nos modelos

A Tabela 2 apresenta os parâmetros utilizados para o estudo da dinâmica populacional do mosquito Aedes aegypti, os valores atribuídos a cada parâmetro neste estudo e suas respectivas fontes. Esses valores estão de acordo com as informações fornecidas pela Dra. Margareth Capurro.

O diagrama da dinâmica considerando os dois controles pode ser visto na Figura 1 e o sistema de equações é dado pelo modelo na sequência. 


\begin{tabular}{|c|c|c|c|}
\hline Notação & Significado & Valor & Fonte \\
\hline$\mu_{H}$ & Taxa de mortalidade de $H$ & 0.0373 & [6] \\
\hline$\mu_{F}$ & Taxa de mortalidade de $F, F_{I}$ e $F_{S}$ & 0.04 & adaptado \\
\hline$\alpha_{F}$ & Taxa de interação entre $F_{S}$ e $I_{H}$ & 0.75 & {$[2]$} \\
\hline$\delta$ & Taxa de inserção dos machos inférteis & livre & - \\
\hline$\mu_{M}$ & Taxa de mortalidade de $M$ & 0.0373 & [6] \\
\hline$\mu_{N}$ & Taxa de mortalidade de $N$ & 0.04 & adaptado \\
\hline$\mu_{G}$ & Taxa de mortalidade de $G_{S}$ e $G_{I}$ & 0.04 & adaptado \\
\hline$\alpha_{G}$ & Taxa de interação entre $G_{S}$ e $I_{H}$ & 0.75 & {$[2]$} \\
\hline$\alpha_{F^{*}}$ & Taxa de interação entre $F_{S}^{*}$ e $I_{H}$ & 0.75 & {$[2]$} \\
\hline$\mu_{F^{*}}$ & Taxa de mortalidade de $F_{I}^{*}$ e $F_{S}^{*}$ & 0.04 & adaptado \\
\hline$\phi_{A}$ & Taxa de oviposição & 4.0479 & [6] \\
\hline$\phi_{A_{E}}$ & Taxa de oviposição do replacement & 4.0479 & [6] \\
\hline$\gamma$ & Taxa de transição entre $A$ e adultos $M$ e $H$ & 0.3706 & [6] \\
\hline$\gamma_{E}$ & Taxa de transição entre $A_{E}$ e adultos $M_{E}$ e $H_{E}$ & 0.3706 & [6] \\
\hline$\mu_{A_{E}}$ & Taxa de mortalidade de $A_{E}$ & 0.1201 & [6] \\
\hline$\mu_{A}$ & Taxa de mortalidade de $A$ & 0.1201 & [6] \\
\hline$\beta$ & Taxa de interação de $M$ e $H$ & 0.7 & {$[5]$} \\
\hline$\beta_{N}$ & Taxa de interação de $N$ e $H$ & 0.5 & [5] \\
\hline$\beta_{E}$ & Taxa de interação de $M$ e $H_{E}$ & 0.7 & {$[5]$} \\
\hline$\beta_{N_{E}}$ & Taxa de interação de $N$ e $H_{E}$ & 0.5 & [5] \\
\hline$\beta_{E^{*}}$ & Taxa de interação de $M_{E}$ e $H_{E}$ & 0.7 & [5] \\
\hline$\mu_{H_{E}}$ & Taxa de mortalidade de $H_{E}$ & 0.0373 & [6] \\
\hline$\mu_{F_{E}}$ & Taxa de mortalidade de $F_{E}$ & 0.04 & adaptado \\
\hline$\alpha_{F_{E}}$ & Taxa de interação entre $F_{E}$ e $I_{H}$ & 0.75 & {$[2]$} \\
\hline$\phi_{H}$ & Taxa de natalidade humana & 0.000457 & {$[3]$} \\
\hline$\sigma_{S}$ & Taxa de interação entre $F_{I}, F_{I}^{*}$ e $S_{H}$ & 0.375 & {$[2]$} \\
\hline$\mu_{S}$ & Taxa de mortalidade de $S_{H}$ & 0.000457 & {$[3]$} \\
\hline$\mu_{I}$ & Taxa de mortalidade de $I_{H}$ & 0.000548 & {$[3]$} \\
\hline$\sigma_{I}$ & Taxa de recuperação dos infectados & 0.3288 & {$[2]$} \\
\hline$\mu_{R}$ & Taxa de mortalidade dos recuperados & 0.000457 & {$[3]$} \\
\hline$r$ & Proporção machos/fêmeas & 0.5 & [6] \\
\hline$K$ & Constante de meio & $10^{6}$ & [6] \\
\hline$K_{H}$ & Constante de meio para humanos & - & - \\
\hline
\end{tabular}

Tabela 2: Parâmetros utilizados nos modelos e respectivas fontes

A população $A(t)$ cresce conforme os ovos são colocados em função de $\phi_{A}$ que é a taxa de oviposição por fêmea fecundada e do termo logístico que controla o crescimento da população (mais especificamente chamamos $K$ de constante do meio), enquanto que essa população decresce conforme os mosquitos se desenvolvem para a fase adulta (taxa de transição $\gamma$ ) e em função da própria taxa de mortalidade da população $\left(\mu_{A}\right)$. A população $M(t)$ cresce a partir da transição entre a fase aquática e a fase adulta de maneira a manter a proporção de machos e fêmeas do meio

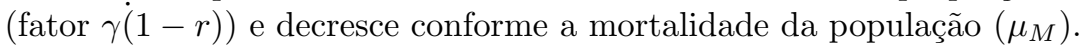

A população $H(t)$ cresce também a partir da transição entre fase aquática e fase adulta e decresce conforme a mortalidade da população $\left(\mu_{H}\right)$ e a fecundação de fêmeas por machos (com a inserção dos mosquitos modificados, ocorre uma competição entre os machos normais e eles para copularem com as fêmeas não fecundadas $H(t)$, e essa competição é modelada pelo fator $M(t)+N(t)+M_{E}(t)$ nos denominadores de $\beta, \beta_{N}$ e $\left.\beta^{*}\right)$. As populações $F_{S}(t), G_{S}(t)$ e $F_{S}^{*}(t)$ aumentam conforme a fecundação das fêmeas em $H(t)$ e decrescem conforme a mortalidade da população $\left(\mu_{F}, \mu_{G}\right.$ e $\left.\mu_{F^{*}}\right)$ e a infecção que se dá pelo contato de fêmeas saudáveis com humanos infectados (fatores $\alpha_{F}, \alpha_{G}$ e $\alpha_{F^{*}}$ ).

Os compartimentos $F_{I}(t), G_{I}(t)$ e $F_{I}^{*}(t)$ crescem conforme a infecção de fêmeas em $F_{S}(t), G_{S}(t)$ e $F_{S}^{*}(t)$ e decrescem com a mortalidade da população $\left(\mu_{F}, \mu_{G}\right.$ e $\left.\mu_{F^{*}}\right)$. Os machos inférteis são colocados a uma taxa constante $\delta$ e a população diminui conforme a mortalidade $\mu_{N}$. 
Note que não precisamos separar as fêmeas que possuem o gene de Replacement em saudáveis e infectadas, pois quando elas se infectam, elas morrem e portanto são desconsideradas na dinâmica. Essas fêmeas, tanto as saudáveis quanto as infectadas, irão gerar descendentes que possuem o gene, por isso, a população aquática que tem o gene aumenta conforme a taxa de oviposição dessas duas populações e da população de fêmeas fecundadas que possuem o gene $F_{E}(t)$, representada pelo fator que acompanha $\phi_{A_{E}}$. Quando as fêmeas não fecundadas que possuem o gene $H_{E}(t)$ são fecundadas, independente do tipo de macho que participa do processo, elas vão para a população de fêmeas fecundadas $F_{E}(t)$ que possuem o gene e dão origem a descendentes com o gene, por isso, mesmo considerando três termos separados na taxa de fecundação de $H_{E}(t)$, representando o contato entre a fêmea não fecundada com os outros machos, ambos os termos vão diretamente para a equação de $F_{E}(t)$ e essa população diminui conforme sua mortalidade e a taxa de infecção.

A dinâmica dos humanos é mais simples de explicar já que a população é dividida em só três compartimentos. A população de humanos saudáveis $S_{H}(t)$ cresce conforme a taxa de natalidade $\left(\phi_{H}\right)$, controlada pelo termo logístico, similar as equações de $A(t)$ e $A_{E}(t)$ e diminui conforme a mortalidade $\left(\mu_{S}\right)$ e a taxa de infecção dessa população, que é dada pelo contato de $F_{I}(t)$, $G_{I}(t)$ e $F_{I}^{*}(t)$ e foi modelada com $\sigma_{S} \cdot\left(F_{I}(t)+G_{I}(t)+F_{I}^{*}(t)\right)$, dividido pelos casos possíveis. A população de humanos infectados cresce conforme a taxa de infecção de humanos saudáveis e decresce com a mortalidade $\left(\mu_{I}\right)$ e a taxa de recuperação dos infectados $\left(\sigma_{I}\right)$. A população dos humanos recuperados cresce conforme a taxa de recuperação dos humanos infectados e decresce com sua mortalidade $\left(\mu_{R}\right)$.
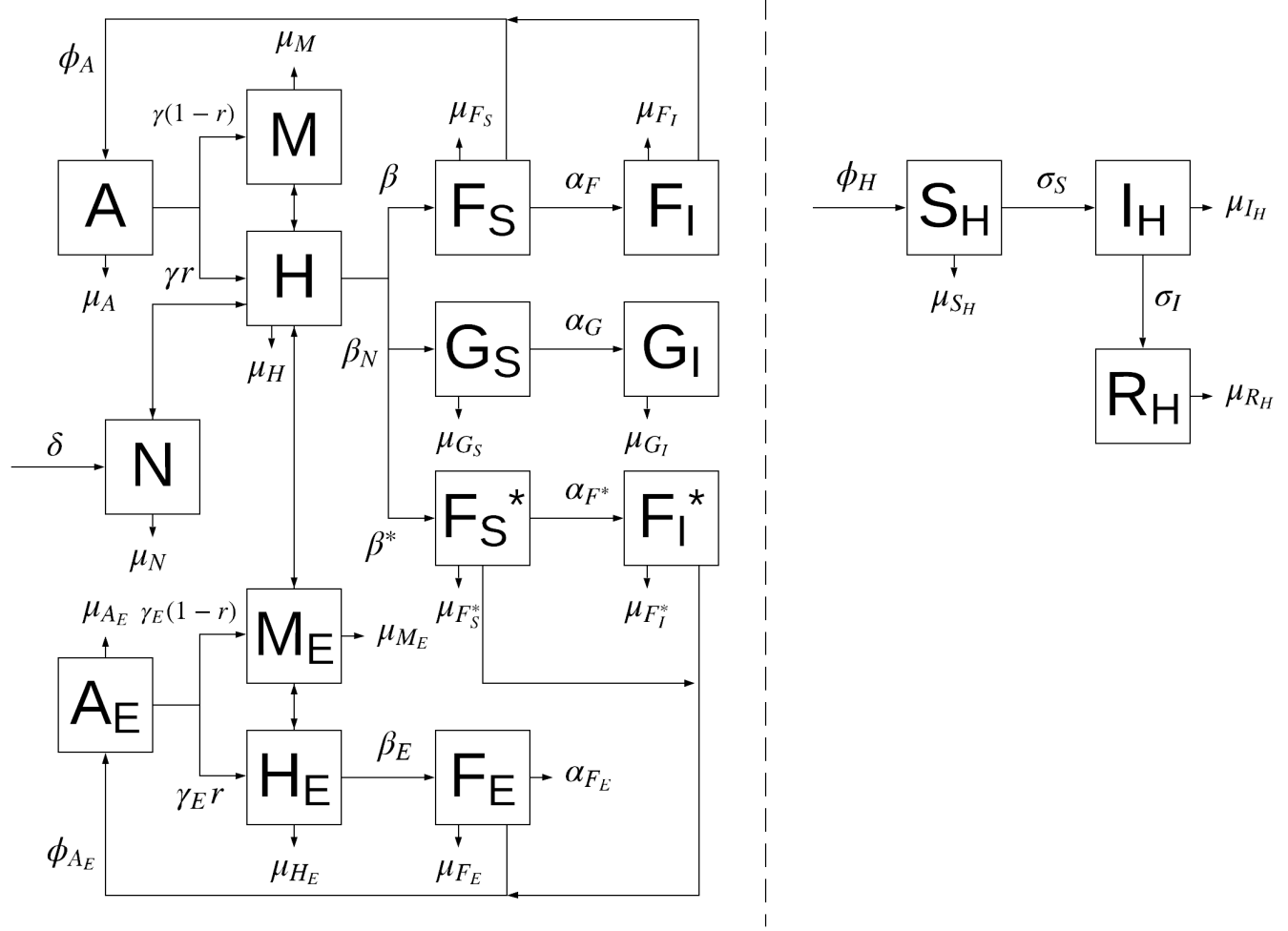

Figura 1: Diagrama representando a dinâmica do modelo com controle SIT e Replacement. 


$$
\begin{aligned}
& \left\{\begin{aligned}
\frac{d A}{d t} & =\phi_{A}\left(1-\frac{A(t)+A_{E}(t)}{K}\right)\left(F_{S}(t)+F_{I}(t)\right)-\left(\gamma+\mu_{A}\right) A(t) \\
\frac{d M}{d t} & =\gamma(1-r) A(t)-\mu_{M} M(t) \\
\frac{d H}{d t} & =\gamma \cdot r A(t)-\beta\left(\frac{M(t) H(t)}{M(t)+N(t)+M_{E}(t)}\right)-\beta_{N}\left(\frac{N(t) H(t)}{M(t)+N(t)+M_{E}(t)}\right)-
\end{aligned}\right. \\
& -\beta^{*}\left(\frac{M_{E}(t) H(t)}{M(t)+N(t)+M_{E}(t)}\right)-\mu_{H} H(t) \\
& \frac{d F_{S}}{d t}=\beta\left(\frac{M(t) H(t)}{M(t)+N(t)+M_{E}(t)}\right)-\mu_{F} F_{S}(t)-\alpha_{F} F_{S}(t)\left(\frac{I_{H}(t)}{S_{H}(t)+I_{H}(t)+R_{H}(t)}\right) \\
& \frac{d F_{I}}{d t}=\alpha_{F} F_{S}(t)\left(\frac{I_{H}(t)}{S_{H}(t)+I_{H}(t)+R_{H}(t)}\right)-\mu_{F} F_{I}(t) \\
& \frac{d N}{d t}=\delta-\mu_{N} N(t) \\
& \frac{d G_{S}}{d t}=\beta_{N}\left(\frac{N(t) H(t)}{M(t)+N(t)+M_{E}(t)}\right)-\mu_{G} G_{S}(t)-\alpha_{G} G_{S}(t)\left(\frac{I_{H}(t)}{S_{H}(t)+I_{H}(t)+R_{H}(t)}\right) \\
& \frac{d G_{I}}{d t}=\alpha_{G} G_{S}(t)\left(\frac{I_{H}(t)}{S_{H}(t)+I_{H}(t)+R_{H}(t)}\right)-\mu_{G} G_{I}(t) \\
& \frac{d F_{S}^{*}}{d t}=\beta^{*}\left(\frac{M_{E}(t) H(t)}{M(t)+N(t)+M_{E}(t)}\right)-\alpha_{F^{*}} F_{S}^{*}(t)\left(\frac{I_{H}(t)}{S_{H}(t)+I_{H}(t)+R_{H}(t)}\right)-\mu_{F^{*}} F_{S}^{*}(t) \\
& \frac{d F_{I}^{*}}{d t}=\alpha_{F^{*}} F_{S}^{*}(t)\left(\frac{I_{H}(t)}{S_{H}(t)+I_{H}(t)+R_{H}(t)}\right)-\mu_{F^{*}} F_{I}^{*}(t) \\
& \frac{d A_{E}}{d t}=\phi_{A_{E}}\left(1-\frac{A(t)+A_{E}(t)}{K}\right)\left(F_{E}(t)+F_{I}^{*}(t)+F_{S}^{*}(t)\right)-\left(\gamma_{E}+\mu_{A_{E}}\right) A_{E}(t) \\
& \frac{d M_{E}}{d t}=\gamma_{E}(1-r) A_{E}(t)-\mu_{M_{E}} M_{E}(t) \\
& \frac{d H_{E}}{d t}=\gamma_{E} \cdot r A_{E}(t)-\beta_{E}\left(\frac{H_{E}(t) M(t)}{M(t)+N(t)+M_{E}(t)}+\frac{H_{E}(t) N(t)}{M(t)+N(t)+M_{E}(t)}+\right. \\
& \left.+\frac{H_{E}(t) M_{E}(t)}{M(t)+N(t)+M_{E}(t)}\right)-\mu_{H_{E}} H_{E}(t) \\
& \frac{d F_{E}}{d t}=\beta_{E}\left(\frac{H_{E}(t) M(t)}{M(t)+N(t)+M_{E}(t)}+\frac{H_{E}(t) N(t)}{M(t)+N(t)+M_{E}(t)}+\frac{H_{E}(t) M_{E}(t)}{M(t)+N(t)+M_{E}(t)}\right)- \\
& -\mu_{F_{E}} F_{E}(t)-\alpha_{F_{E}} F_{E}(t)\left(\frac{I_{H}(t)}{S_{H}(t)+I_{H}(t)+R_{H}(t)}\right)
\end{aligned}
$$




$$
\left\{\begin{array}{l}
\frac{d S_{H}}{d t}=\phi_{H} \cdot\left(1-\frac{S_{H}(t)+I_{H}(t)+R_{H}(t)}{K_{H}}\right)\left(S_{H}(t)+I_{H}(t)+R_{H}(t)\right)- \\
-\sigma_{S}\left(F_{I}(t)+G_{I}(t)+F_{I}^{*}(t)\right) \frac{S_{H}(t)}{S_{H}(t)+I_{H}(t)+R_{H}(t)}-\mu_{S_{H}} S_{H}(t) \\
\frac{d I_{H}}{d t}=\sigma_{S}\left(F_{I}(t)+G_{I}(t)+F_{I}^{*}(t)\right) \frac{S_{H}(t)}{S_{H}(t)+I_{H}(t)+R_{H}(t)}-\left(\mu_{I_{H}}+\sigma_{I}\right) I_{H}(t) \\
\frac{d R_{H}}{d t}=\sigma_{I} I_{H}(t)-\mu_{R_{H}} R_{H}(t)
\end{array}\right.
$$

\section{Resultados}

Nesta Seção, apresentamos os resultados e uma breve análise das simulações computacionais. Para a implementação computacional foi utilizada a linguagem Python e foi usado o Método de Euler para resolução dos sistemas de equações diferenciais ordinárias (EDOs).

Consideramos neste trabalho a dinâmica populacional em uma cidade com 1 milhão de habitantes. A Tabela 3 mostra os dados das populações consideradas nesta simulação.

\begin{tabular}{llll}
\hline Notação & \multicolumn{1}{c}{ Descrição } & Pop. Inicial & Break Point \\
\hline$A$ & Fase aquática & 10000000 & 67944908 \\
$M$ & Machos selvagens & 500000 & 27223221 \\
$H$ & Fêmeas saudáveis não fecundadas & 500000 & 10881719 \\
$F_{S}$ & Fêmeas saudáveis fecundadas por machos selvagens & 0 & 16252626 \\
$F_{I}$ & Fêmeas infectadas fecundadas por machos selvagens & 0 & 1569 \\
$A_{E}$ & Fase aquática replacement & 0 & $0(100000)$ \\
$S_{H}$ & Humanos suscetíveis & 1000000 & 999526 \\
$I_{H}$ & Humanos infectados & 1 & 290 \\
$R_{H}$ & Humanos recuperados & 0 & 5 \\
\hline
\end{tabular}

Tabela 3: Dados das populações consideradas na Simulação III

O modelo foi executado e observamos indícios de que um surto de dengue pode ocorrer nessa população, mesmo que se inicie com apenas uma pessoa infectada, conforme resultados obtidos ao final da simulação do modelo básico por 7 dias, apresentados na coluna 'Break Point' da Tabela 3. Após 7 dias, introduzimos os métodos de combate na simulação: inserção diária de 1000 machos SIT e liberação única na natureza de cem mil ovos geneticamente modificados que geram descendentes com o gene replacement (dado entre parênteses na tabela). A Figura 2 apresenta os resultados.

A estratégia se mostrou eficiente no combate à doença, já que, como podemos ver na Figura 2 , a população antiga de mosquitos fêmeas normais é erradicada e, consequentemente, a doença também. Essa população selvagem é substituída após cerca de 9 anos de simulação. Vale ressaltar que o tempo necessário para isso foi quase o mesmo da simulação na qual utilizamos apenas o controle do replacement. Utilizar as duas estratégias combinadas não gerou uma diferença significativa nos resultados. Também observou-se que a população de humanos infectados diminuiu muito rapidamente na simulação mesmo antes da população de fêmeas nativas ser controlada, o que pode ser explicado por se tratar de um modelo simplificado de SIR no qual uma pessoa se torna imune uma vez recuperada, assim, depois de um surto os casos acabam ficando controlados. 

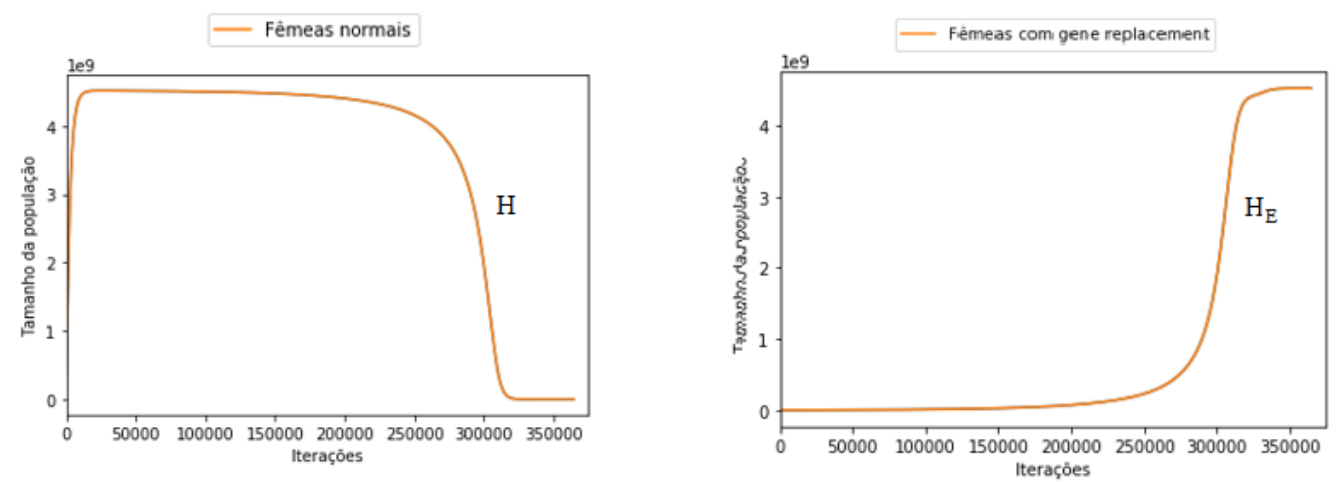

Figura 2: Simulação III - Representação gráfica do crescimento das diferentes populações em função do tempo usando controle SIT e estratégia de replacement.

\section{Conclusões}

Neste trabalho, foi realizado um estudo da dinâmica populacional do mosquito Aedes aegypti sob duas estratégias de controle. O controle SIT, em que machos infeŕteis são inseridos na população, e a estratégia de replacement, ou substituição, foram investigados separadamente e em conjunto. No controle por replacement, mosquitos transgênicos que morrem ao contrair o vírus e, portanto, não são transmissores da doença, são inseridos no meio ambiente para transmitir tal característica aos seus descendentes, de forma a substituir gradualmente a população selvagem.

As relações dos diversos grupos populacionais entre si e com o meio foram descritas matematicamente através de sistemas de equações diferenciais. A evolução de cada grupo populacional ao longo do tempo foi obtida por meio da resolução numérica dos sistemas de EDOs, utilizando o Método de Euler. O objetivo deste estudo foi permitir um melhor entendimento dos efeitos de cada controle e trazer insights que apoiassem a tomada de decisão para adoção da melhor estratégia de combate à transmissão da dengue.

O estudo da dinâmica populacional com controle através da modificação genética para replacement ainda não foi explorado na literatura, de forma que este trabalho traz uma importante contribuição sobre o tema e pode ser visto como uma abordagem inicial do problema. Para trabalhos posteriores, sugere-se uma análise mais aprofundada dos resultados em relação a pontos de equilíbrio, estabilidade e evolução temporal das populações.

Os resultados obtidos foram considerados promissores, mas tem-se em vista que são necessárias modificações para que o modelo possa se adequar cada vez mais a realidade. Sendo mais simples, o modelo SIR não é considerado o melhor para tratar de transmissão de doenças como a dengue e não reflete com precisão a situação real. Além disso, os parâmetros de calibração usados refletem estimativas de dados nem sempre precisas. Considerado isto, o trabalho tem potencial de auxiliar em estudos futuros mais detalhados sobre o tema.

\section{Referências}

[1] Bartlett, A. C., and Staten, R. T. The sterile insect release method and other genetic control strategies. Radcliffe's IPM world textbook, 1996.

[2] Braselton, J. P., and Bakach, I. A survey of mathematical models of dengue fever. Journal of Computer Science Systems Biology, 8(5), 255, 2015. 
[3] Kuniyoshi, M. L. G., and dos Santos, F. L. P. Mathematical modelling of vector-borne diseases and insecticide resistance evolution. Journal of Venomous Animals and Toxins including Tropical Diseases, 23(1), 34, 2017.

[4] Ministério da Saúde, Boletim Epidemiológico n⿳0 02/2020 Aedes (dengue, chikungunya e zika) de 29/12/2019 a 01/02/2020. Acesso em 13 de Março de 2020, 2020.

[5] Thomé, R. C., Yang, H. M., and Esteva, L. Optimal control of Aedes aegypti mosquitoes by the sterile insect technique and insecticide. Mathematical Biosciences, 223(1), 12-23, 2010.

[6] Yang, H. M., Boldrini, J. L., Fassoni, A. C., Freitas, L. F. S., Gomez, M. C., de Lima, K. K. B., and Freitas, A. R. R. Fitting the incidence data from the city of Campinas, Brazil, based on dengue transmission modellings considering time-dependent entomological parameters. PloS one, $11(3), 2016$. 